\title{
Current status of LEGEND: Searching for Neutrinoless Double-Beta Decay in ${ }^{76} \mathrm{Ge}$ : Part I
}

\author{
I.Guinn * \\ University of North Carolina \\ E-mail: iguinn@uw.edu \\ J.M.López-Castaño ${ }^{\dagger}$ \\ University of South Dakota \\ E-mail: Mariano.Lopez@usd.edu
}

\begin{abstract}
Neutrinoless double-beta decay $(0 v \beta \beta)$ decay is a hypothetical process that violates lepton number, and whose observation would unambiguously indicate that neutrinos are Majorana fermions. In the standard inverted-ordering neutrino mass scenario, the minimum possible value of $\mathrm{m}_{\beta \beta}$ corresponds to a half-life around $10^{28} \mathrm{yr}$ for $0 v \beta \beta$ decay in ${ }^{76} \mathrm{Ge}$, which is the target of the next generation of experiments. The current limits of GERDA and MAJORAnA DEMOnSTRATOR indicate a half-life higher than $10^{26} \mathrm{yr}$. These experiments use high-purity germanium (HPGe) detectors that are highly-enriched in ${ }^{76} \mathrm{Ge}$. They have achieved the best intrinsic energy resolution and the lowest background rate in the signal search region among all $0 v \beta \beta$ experiments.

Taking advantage of these successes, a new international collaboration - the Large Enriched Germanium Experiment for Neutrinoless $\beta \beta$ Decay (LEGEND) - has been formed to build a tonscale experiment with discovery potential covering the inverse-ordering neutrino mass range in a decade, following a phased approach. This first part of LEGEND proceedings describes GERDA and MAJORAnA DEMONSTRATOR capabilities and the general plan of LEGEND to reach the goal, while the second part is focused in the status of the first stage of LEGEND, LEGEND-200.
\end{abstract}

XXIX International Symposium on Lepton Photon Interactions at High Energies - LeptonPhoton2019 August 5-10, 2019

Toronto, Canada

*Speaker of "LEGEND: Searching for Neutrinoless Double-Beta Decay in ${ }^{76} \mathrm{Ge}$ ".
${ }^{\dagger}$ Speaker of "Current Status of LEGEND". 

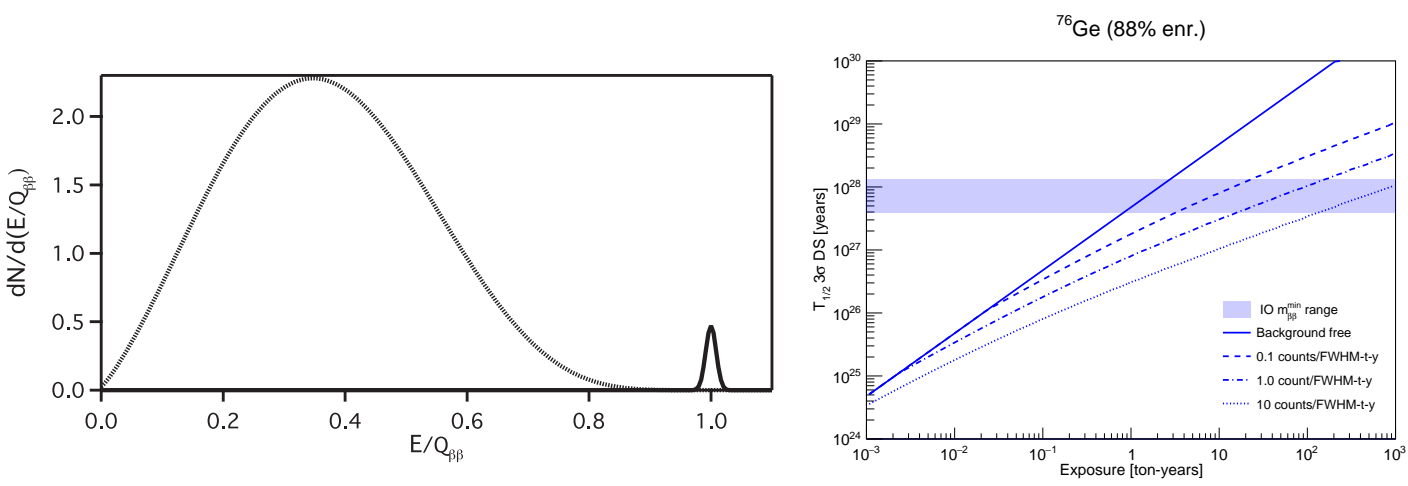

Figure 1: Left: a simulated $0 v \beta \beta$-decay electron energy spectrum. The broad spectrum comes from $2 v \beta \beta$ while the peak at the endpoint comes from $0 v \beta \beta$, with a branching ratio of $1 \%$. Right: Discovery potential at $3 \sigma$ confidence for $0 v \beta \beta$ as a function of isotopic exposure and background index. The blue band represents the range of half-lives corresponding to the $17 \mathrm{meV}$.[1]

\section{Introduction}

Double beta decay $(2 v \beta \beta)$ is a process in which two neutrons change into two protons emitting two electrons and two anti-neutrinos, and has been observed in several isotopes. Neutrinoless double beta decay $(0 v \beta \beta)$ decay is a similar process in which the anti-neutrinos are not emmited: $X_{Z}^{N} \rightarrow Y_{Z+2}^{N}+2 e^{-}$. The signature of $0 v \beta \beta$ decay is the emission of two electrons with total energy corresponding to the decay Q-value, and its half-life $\left(T_{1 / 2}^{0 v}\right)$ is proportional to the effective neutrino mass $\left(m_{\beta \beta}\right)$. The goal for the next generation of $0 v \beta \beta$ experiments is a sensitivity to $m_{\beta \beta}$ of $\sim 17 \mathrm{meV}$, which cover all possible values in the inverted-ordering neutrino mass scenario [2].

${ }^{76} \mathrm{Ge}$ undergoes $2 v \beta \beta$, and several experiments are actively searching for $0 v \beta \beta$ in ${ }^{76} \mathrm{Ge}$. These experiments utilize High-Purity Germanium (HPGe) detectors which are enriched to at least $85 \%$ in ${ }^{76} \mathrm{Ge}$, meaning the ${ }^{76} \mathrm{Ge}$ acts as both source and detector of $0 v \beta \beta$. Different variations of semi-coaxial or P-type Point Contact (PPC) detector geometries are used, because they provide advantages in energy resolution and background discrimination over planar and coaxial ones. Achieving a sensitivity to $17 \mathrm{meV}$ of $m_{\beta \beta}$ requires a half-life sensitivity of $10^{28} \mathrm{y}$, which can be achieved with $\sim 10$ tonne-y of exposure with $<0.1 \mathrm{cts} /$ FWHM-t-y of backgrounds in the region of interest for $0 v \beta \beta$, as shown in Figure 1.

\subsection{GERDA}

GERDA [3], shown in Figure 2, is located in the Laboratori Nazionali del Gran Sasso (LNGS). It utilizes $15.6 \mathrm{~kg}$ of semi-coaxial detectors and $20 \mathrm{~kg}$ of Broad Energy Germanium (BEGe) detectors. To achieve low background, the detectors are immersed in liquid argon (LAr), which acts as a scintillating active veto. GERDA has collected $46.7 \mathrm{~kg}$-y of isotopic exposure, with a background index of $2.6 \mathrm{cts} / \mathrm{FWHM}$-t-y for the BEGe detectors. GERDA has achieved a median $T_{1 / 2}^{0 v}$ sensitivity of $5.8 \times 10^{25} \mathrm{y}$ and $90 \% \mathrm{CL}$ limit of $8 \times 10^{25} \mathrm{yr}$ [3]. These results represent the lowest background index (BI) and highest half-life sensitivity of any currently operating $0 v \beta \beta$ experiment. 

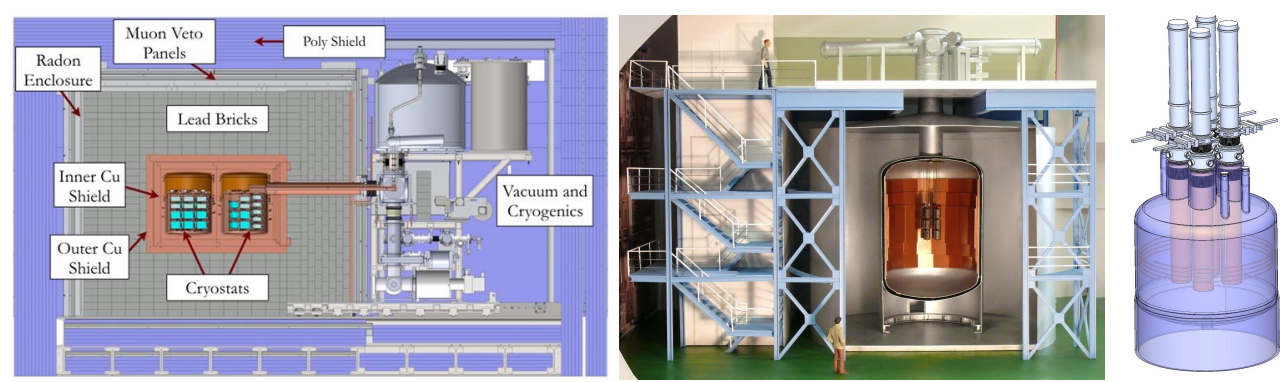

Figure 2: Left: A drawing of the Majorana Demonstrator experiment. Middle: A drawing of the GERDA experiment. The LEGEND-200 experiment is repurposing this infrastructure for a larger detector array. Right: A baseline design for LEGEND-1000.

\subsection{Majorana Demonstrator}

The Majorana Demonstrator (MJD) [4], shown in figure 2, is operating $44.8 \mathrm{~kg}$ of PPC HPGe detectors in vacuum at the Sanford Underground Research Facility (SURF). $29.7 \mathrm{~kg}$ of the detectors are enriched to $88 \%$ in ${ }^{76} \mathrm{Ge}$, while remainder are natural isotopic abundance BEGe detectors. MJD uses ultra-clean materials in its construction, including copper that was electroformed underground to minimize cosmogenic activation and specially designed cables, connectors and low-noise electronics. The enriched detectors have achieved a world-leading energy resolution of $2.5 \mathrm{keV}$ at the $2039 \mathrm{keV}$ Q-value $(0.12 \%)$. MJD has collected $26 \mathrm{~kg}$-y of isotopic exposure with a BI of $4.0 \mathrm{cts} / \mathrm{FWHM}-\mathrm{t}-\mathrm{y}$, and has established a $T_{1 / 2}^{0 v}$ limit of $2.6 \times 10^{25} \mathrm{y}$ at $90 \%$ CL[4].

\section{LEGEND}

LEGEND aims to explore a region of $0 v \beta \beta$ half-life in ${ }^{76} \mathrm{Ge}$ improved by 2 orders of magnitude, from $10^{26} \mathrm{yr}$ to $10^{28} \mathrm{yr}[5]$. To achieve this, LEGEND will combine the best technologies of GERDA and the MAJORANA DEMOnSTRATOR. LEGEND will be operated in two phases. The first phase, LEGEND-200, is currently being constructed as a $200 \mathrm{~kg}$ detector array with a background goal of $<0.6 \mathrm{cts} / \mathrm{FWHM}$-t-y and is expected to begin operation at LNGS in 2021.

LEGEND-1000 is the next phase of LEGEND, currently in the design stage, that will consist of a $\sim 1$ tonne array of HPGe detectors immersed in LAr. Active R\&D efforts are underway to achieve a further reduction in backgrounds to $<0.1 \mathrm{cts} / \mathrm{FWHM}-\mathrm{t}-\mathrm{y}$. These include the development of larger HPGe detectors, optimized LAr light collection, improvements in clean materials and handling, and the use of depleted argon from underground sources.

\section{References}

[1] S.R. Elliot. Talk on APS april Meeting 2019.

[2] M. Agostini, G. Benato and J. Detwiler. Phys. Rev. D 96, no.5, 053001 (2017)

[3] GERDA Collaboration. Science, eaav-8613 (2019)

[4] MAJORANA Collaboration. Phys. Rev. C 100025501 (2019)

[5] LEGEND Collaboration. AIP Conf. Proc. 1894, 020027 (2017) 